\title{
Highly Precise Micropositioning Task using a Direct Visual Servoing Scheme
}

\author{
B. Tamadazte, G. Duceux, N. Le-Fort Piat, and E. Marchand,
}

\begin{abstract}
This paper demonstrates accurate micropositioning scheme based on a direct visual servoing process. This technique uses only the pure image signal (photometric information) to design the control law. With respect to traditional visual servoing approaches that use geometric visual features (points, lines ...), the visual features used in the control law are the pixel intensity. The proposed approach was tested in term of accuracy and robustness in several experimental conditions. The obtained results have demonstrated a good behavior of the control law and very good positioning accuracy. The obtained accuracies are estimated to $14 \mathrm{~nm}, 89 \mathrm{~nm}$, and 0.001 degrees in the $x, y$ and $\theta$ axes of positioning platform, respectively.
\end{abstract}

\section{OVERVIEW}

The heterogeneous integration of high-performance electronic devices, microelectromechanical structures (MEMS), and optoelectronic devices onto the same substrate is important for the development of low-cost, and high-compact microsystems [9]. To set-up compact microsystems, it is necessary to be able to handle and assemble the various elements that constitute these microsystems. These operations (i.e. handling and assembling) must be achieved with high accuracy. In the last decade considerable researches have been performed on the development of robotic microassembly station, gripping systems, precise actuators, and microassembly strategies. Many works have dealt with the development of control approaches to automate the different micromanipulation and microassembly tasks such positioning, orientation, picking, placing, and insertion of the different micro-objects [10], [8]. Most of these works considered the use of the vision system to control the behavior of the robotic structure of the microassembly station during the assembly process. Thus, the control of robots through realtime and continuous visual feedback is generally known as visual servoing [4], and the continuous observation of the objects of interest is referred to visual tracking [11], [10]. Visual tracking of an object involves the detection of some known object features in the acquired images and, using these features, it is possible to estimate the the object position and orientation. A feature can be a distinctive part of the object and can exist naturally as a part of the geometry (i.e.

This work is partially conducted with financial support from the project "Hybrid Ultra Precision Manufacturing Process Based on Positional and Self assembly for Complex Micro-Products (HYDROMEL NMP2-CT-2006026622)" funded by the European Commission.

B. Tamadazte, G. Duceux, and N. Le-Fort Piat are with the FEMTOST Institute, UMR CNRS 6174-UFC/ENSMM/UTBM. Automatic Control and Micro-Mechatronic Systems Department (AS2M). 24 rue Alain Savary, 25000 Besançon, France. brahim.tamadaztedens $2 \mathrm{~m}$. fr

E. Marchand is with Université de Rennes 1, IRISA, INRIA RennesBretagne Atlantique, Lagadic research group, Rennes 35042, France. corners, or edges), or as a deliberately fabricated marking on the surface (i.e. markers). This tracking process is one of the bottlenecks of the development of visual servoing techniques. Recently, it has been shown that these tracking and matching processes can be totally removed and that no other information than the image intensity [7], [2], the image entropy [3], or the image Laplacian can be considered to control a robot. Considering that the control of robot is directly linked to the variation of the pixels intensity in the image, no geometric information has to be extracted and, consequently, tracked or matched over time. This avoid the development of complex visual tracking algorithms such as in [10], [11]. Let us also advocate that avoiding this feature extraction process allows to be naturally robust to error measurements increasing accuracy and repeatability of the positioning tasks. This accuracy is also substantially increased since highly redundant information (all the pixels of the image) is considered to design the control law. In this paper, we will consider such a direct approach to control the microrobot motions with high accuracy. We consider a photometric visual servoing approach since lighting conditions are constant. Despite the fact that no complex image processing algorithms are considered, we will show that these new techniques are robust to different perturbations such as the occlusion or addition of others micro-objects during the manipulation process.

Section II illustrates MEMS microassembly station which consists of a 5 DOF microassembly workcell equipped with a top view optical video microscope, and a semi-controlled environment. Section III presents some definitions about the principles of the eye-to-hand visual servoing. Section IV describes the new direct visual servoing approach using only the pixels intensity of the image as visual features, and the design of the new control law. Section V discusses the experimental results using the developed method in terms of obtained accuracy quality during the different positioning and orientation tasks, and in different experimental conditions. The microscope magnification will also be controlled using the proposed approach.

\section{EXPERIMENTAL SETUP}

The integration of the developed concept is done on the microassembly workcell illustrated in Figure 1. This MEMS microassembly station had been developed in our laboratory. It includes 3 DOF positioning platform with two linear stages $x y$ and one rotating stage $\theta$. The characteristics of these stages are summarized in Table I. The imaging system is a video stereomicroscope of the type LEICA MZ $16 \mathrm{~A}$. It 


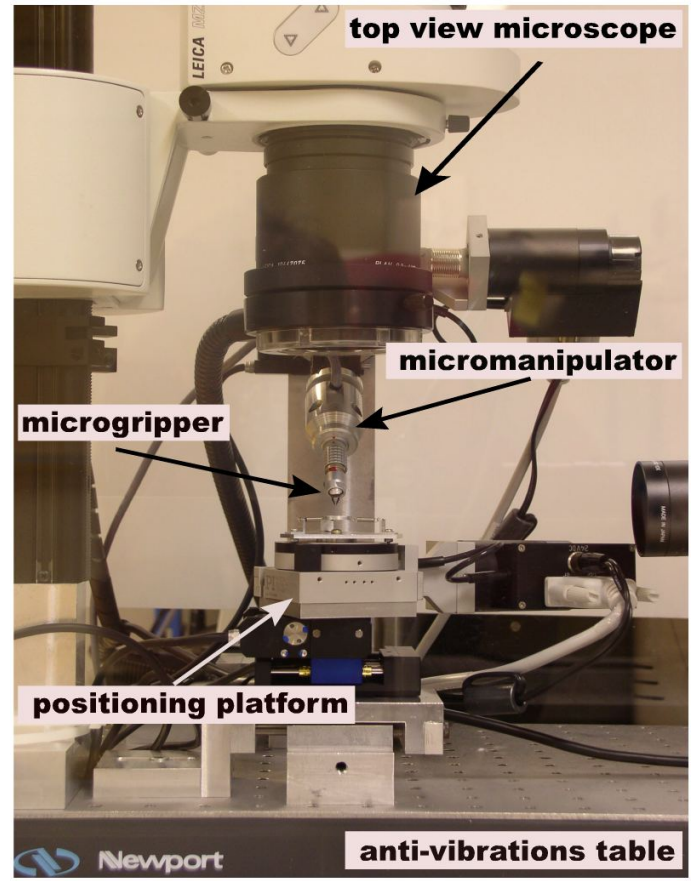

Fig. 1. Photography of the micromanipulation workcell.

delivers a top view images of $1024 \times 772$ pixels of the work scene and it is equipped with a direct illumination source placed around the objective-lens. The zoom (and thus the magnification) and the focus are motorized and controlled by a PC. The Field-of-View varies from $700 \mu \mathrm{m} \times$ $900 \mu \mathrm{m}$ (with a resolution of $1.4 \mu \mathrm{m} / \mathrm{pixel}$ ) at the maximum magnification, to $20 \mathrm{~mm} \times 25 \mathrm{~mm}$ (with a resolution of $21 \mu \mathrm{m} / \mathrm{pixel}$ ) at the minimum magnification. The Depth-ofField varies from $2.9 \mathrm{~mm}$ to $0.035 \mathrm{~mm}$ and the working distance is approximately $130 \mathrm{~mm}$. This set-up is placed on an anti-vibrations table and inside a controlled environment.

TABLE I

LINEAR AND ANGULAR STAGES SPECIFICATIONS.

\begin{tabular}{ll}
\hline \hline & \multicolumn{2}{c}{ Linear motion stages } \\
\hline resolution & $0.007 \mu \mathrm{m}$ \\
increment & $0.05 \mu \mathrm{m}$ \\
max velocity & $1.5 \mathrm{~mm} / \mathrm{s}$ \\
stroke & $25 \mathrm{~mm}$ \\
\hline \multicolumn{2}{c}{ Angular motion stages } \\
\hline resolution & $26 \mu \mathrm{rad}$ \\
increment & $26 \mu \mathrm{rad}$ \\
max velocity & $0.78 \mathrm{rad} / \mathrm{s}$ \\
\hline \hline
\end{tabular}

\section{Traditional Visual Servoing Approaches}

Visual servoing techniques consist of using the data provided by one or several cameras in order to the control the motion of a robotic system [1], [6]. Whatever, the sensor configuration a set of visual features $\mathbf{s}$ has to be designed from the visual measurements $\mathbf{x}(t)(\mathbf{s}=\mathbf{s}(\mathbf{x}(t)))$, allowing control of the desired DOF. A control law has to be designed also so that these features $\mathbf{s}$ reach a desired value $\mathbf{s}^{*}$, defining a correct realization of the task. The aim of vision-based control schemes [6] is to minimize the error e typically defined by:

$$
\mathbf{e}=\mathbf{s}-\mathbf{s}^{*}
$$

The equation that links the variation $\dot{\mathbf{s}}$ of the visual features $\mathbf{s}$ to the robot instantaneous camera velocity $\mathbf{v}$ ( $\mathbf{v}=(\boldsymbol{v}, \boldsymbol{\omega})$ with $\boldsymbol{v}$ the instantaneous linear velocity and $\boldsymbol{\omega}$ the instantaneous angular velocity) is given by:

$$
\dot{\mathbf{s}}=\mathbf{L}_{\mathbf{s}} \mathbf{V}
$$

where $\mathbf{L}_{\mathrm{s}}$ represents the interaction matrix that relates to $\mathbf{s}$ and $\mathbf{v}$, the robot instantaneous velocity [1]. If we want to control the robot using the joint velocities, we have:

$$
\dot{\mathbf{s}}=\mathbf{J}_{\mathbf{s}} \dot{\mathbf{q}}
$$

where $\mathbf{J}_{\mathbf{s}}$ is the features Jacobian and $\dot{\mathbf{q}}$ the joint velocities. In this paper, we mainly consider an eye-to-hand configuration. We then have:

$$
\mathbf{J}_{\mathbf{s}}=-\mathbf{L}_{\mathbf{s}}{ }^{c} \mathbf{V}_{\mathcal{F}}^{\mathcal{F}} \mathbf{J}_{n}(\mathbf{q})
$$

where

- ${ }^{\mathcal{F}} \mathbf{J}_{n}(\mathbf{q})$ is the robot Jacobian expressed in the robot reference frame $R_{\mathcal{F}}$. In our case, the robot Jacobian is computed for the 3 DOF positioning platform (see, Fig. 1) used to validate the proposed approach.

- ${ }^{c} \mathbf{V}_{\mathcal{F}}$ transforms the velocity screw between coordinate frames (here the camera frame $R_{c}$ and the robot reference frame $R_{\mathcal{F}}$ ).

Thereafter, the control law is designed as follows:

$$
\dot{\mathbf{q}}=-\lambda \mathbf{J}_{\mathbf{s}}^{+} \mathbf{e}
$$

where $\lambda$ is the proportional coefficient involved in the exponential convergence of the error, and $\mathbf{J}_{\mathbf{S}}^{+}$the pseudoinverse of $\mathbf{J}_{\mathbf{s}}$.

\section{Visual Servoing Without Explicit Feature EXTRACTION}

In traditional visual servoing techniques, the visual features $\mathbf{s}$ are built from image measurements. $\mathbf{s}$ are mainly geometric features such as points or lines coordinates, image moments or even 3D features. These classical approaches require detection, tracking or matching steps and an estimation in each frame of the value of the visual features. This tracking or matching process has been, to date, considered as a necessary step and is also one of the bottlenecks limiting the expansion of visual servoing.

In this section, we shall consider a direct visual control scheme. The proposed method uses the pure image signal to design the vision-based control law. Rather than computing the visual from some image measurements, we consider that the vector of the visual features is the image itself. The visual features $\mathbf{s}$ are replaced by the image pixels intensity I. Therefore, we can write:

$$
\mathbf{s}=\mathbf{I}
$$


In our case, the image's dimension is $1024 \times 772$ pixels. I can be also write in the following form:

$$
\mathbf{I}=\left(\mathbf{I}_{1} \bullet, \mathbf{I}_{2 \bullet}, \cdots, \mathbf{I}_{\mathrm{N} \bullet}\right)
$$

where $\mathbf{I}_{k} \bullet$ is nothing but the $k$-th line of the image and $\mathbf{N}$ is the number of the lines in the image. To design the controller, we have to consider the interaction matrix that links the variation of the image intensity to the camera velocity [2], [7].

Considering the Optical Flow Constraint Equation (OFCE) hypothesis [5], we can compute the interaction matrix that links the variation of a pixel intensity to the camera motion. The OFCE states that the intensity $\mathbf{I}(\mathbf{x}, t)$ of each projected physical point in the image remains the same during a short time interval $d t$. We have:

$$
\mathbf{I}(\mathbf{x}, t)=\mathbf{I}(\mathbf{x}+\mathbf{d x}, t+d t)
$$

A first order Taylor expansion of the equation (8) gives:

$$
\frac{\partial \mathbf{I}}{\partial x} d x+\frac{\partial \mathbf{I}}{\partial y} d y+\frac{\partial \mathbf{I}}{\partial t} d t=0
$$

which can be written as follows:

$$
\dot{\mathbf{I}}=-\nabla \mathbf{I}_{x} \dot{x}-\nabla \mathbf{I}_{y} \dot{y}
$$

with

$$
\nabla \mathbf{I}_{x}=\frac{\partial \mathbf{I}}{\partial x}
$$

and

$$
\nabla \mathbf{I}_{y}=\frac{\partial \mathbf{I}}{\partial y}
$$

Now, the temporal variations of the image must be linked to the camera displacements. For this, we introduce the interaction matrix of a point of the image which links the point velocity in the image to the camera velocity [1]. It is given by:

$$
\dot{x}=\mathbf{L}_{x} \mathbf{v}
$$

and

$$
\dot{y}=\mathbf{L}_{y} \mathbf{v}
$$

where $\mathbf{L}_{x}$ and $\mathbf{L}_{y}$ are the interaction related to the point:

$$
\begin{aligned}
& \mathbf{L}_{x}=\left(\begin{array}{llllll}
-1 / Z & 0 & x / Z & x y & -(1+x) & y
\end{array}\right.
\end{aligned}
$$

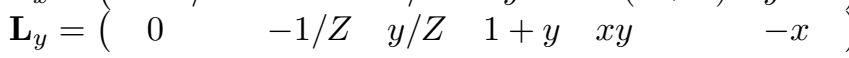

Hence, introducing equations (13) and (14) in the equation (10), we obtain:

$$
\dot{\mathbf{I}}=-\left(\nabla \mathbf{I}_{x} \mathbf{L}_{x}+\nabla \mathbf{I}_{y} \mathbf{L}_{y}\right) \mathbf{v}
$$

or

$$
\dot{\mathbf{I}}=\mathbf{L}_{\mathbf{I}} \mathbf{V}
$$

Knowing the interaction matrix $\mathbf{L}_{\mathbf{I}}$, it is possible to design a control law. As in [2], we use a control law inspired from the Levenberg-Maquardt optimization algorithm. This provides an efficient numerical solution to the problem of minimizing the error function $\mathbf{I}-\mathbf{I}^{*}$ which is highly non-linear. It is the interpolation of the Gauss-Newton and gradient descent method. More stable than a simple gradient descent, it converges faster that the Gauss-Newton scheme corresponding to equation (5). Therefore, the platform velocity $\dot{\mathbf{q}}$ is given by:

$$
\dot{\mathbf{q}}=-\lambda(\mathbf{H}+\mu \cdot \operatorname{diag}(\mathbf{H}))^{-1} \mathbf{J}_{\mathbf{I}}^{\top}\left(\mathbf{I}-\mathbf{I}^{*}\right)
$$

where $\mathbf{J}_{\mathbf{I}}$ represents the Jacobian matrix computed from interaction matrix (16) and (4) computed at the desired position. The parameters $\lambda$ and $\mu$ are positive gains and $\operatorname{diag}(\mathbf{H})$ is the matrix of diagonal terms of the combination matrix $\mathbf{H}$ which is given by:

$$
\mathbf{H}=\mathbf{J}_{\mathbf{I}}^{\top} \mathbf{J}_{\mathbf{I}}
$$

To improve the convergence rate, we implemented an adaptive gain $\lambda$ (the gain increases when the error decreases). The parameter $\lambda$ is a function of the difference between the current image $\mathbf{I}$ and the desired image $\mathbf{I}^{*}$. It is given by:

$$
\lambda=\beta\left\|\mathbf{I}-\overline{\mathbf{I}^{*}}\right\|^{2}
$$

where $\beta$ is a positive gain which allows to adjust the initial value of $\lambda$ (in the presented experiments, $\beta$ have a value of $10^{2} / M, M$ being the number of pixels in the image).

\section{ExPERIMENTAL RESUlts}

\section{A. Validation in Nominal Conditions}
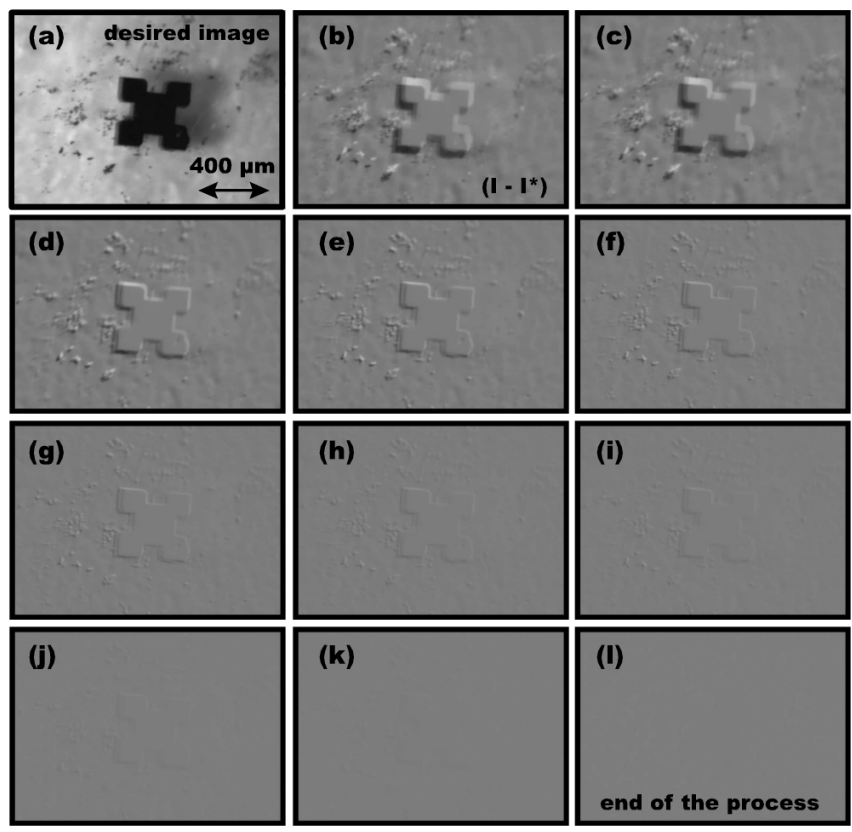

Fig. 2. (a) represents the initial position of the micro-object, (b) to (k) show the error $\left(\mathbf{I}-\mathbf{I}^{*}\right)$ between the current image $\mathbf{I}$ and the desired image $\mathbf{I}^{*}$, and (i) shows the end of the visual servoing process where $\mathbf{I}-\mathbf{I}^{*}=0$.

The proposed method has been validated on our microassembly workcell. The task studied in this paper concerns the automatic positioning of silicon micro-objects. Object's dimensions are range from 300 to 600 micrometers. In the first experiment, only 3 DOF of the positioning platform are controlled (i.e. planar positioning). In the second 
experiment, the controller of the optical microscope magnification (zoom) has been included in the visual servoing scheme. Various experimental situations were tested considering additional external disturbances (i.e. light changing, adding other micro-object) during the positioning process.

The first experiment concerns the positioning of a microobject with dimensions of $400 \times 400 \times 100 \mu \mathrm{m}^{3}$. Figure 2 illustrates a sequence of images of the scene acquired during the positioning task. Figure 2(a) shows the initial position of the micro-object, and Figures 2(b) to (k) represent the error between the acquired image (current image) and the desired position (reference image) i.e. I - I*. Figure 2(l) shows the error at end of the positioning task.

Figure 3 shows the joint velocities (on the 3 DOF $x y \theta$ ) of the positioning platform during the positioning process. It can be seen the good convergence behavior of the proposed control law. In this Figure, abscissa unit is iteration. Considering that we have a close-loop system, an iteration correspond to one step of the control scheme (it includes image processing and the computation of the control law). Considering that the image acquisition rate is 25 frames per second, each iteration lasts 0.040 second. The complete positioning has been achieved in 5.2 seconds.

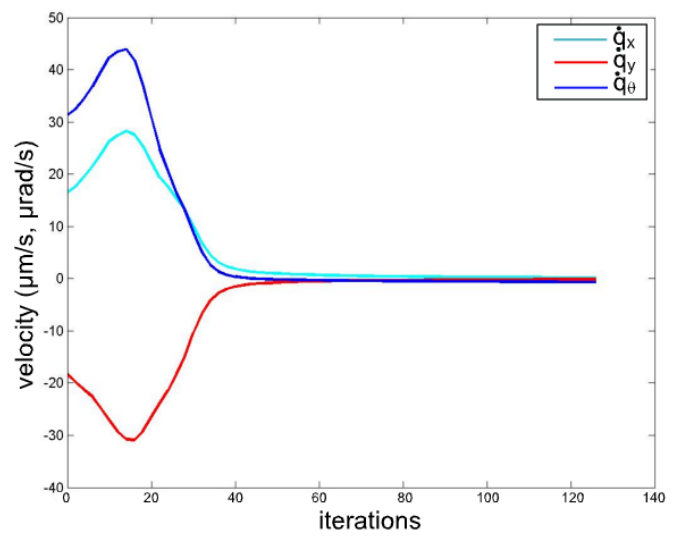

Fig. 3. Joint velocities components (in $\mu \mathrm{m} / \mathrm{s}$ and $\mu \mathrm{rad} / \mathrm{s}$ ) at each iteration of control scheme $\left(\mathbf{q}_{\mathbf{x}}, \mathbf{q}_{\mathbf{y}}\right.$ and $\left.\dot{q}_{\theta}\right)$ for the first experiment shown on Figure 2.

The obtained accuracies in the presented experiment are estimated to $89 \mathrm{~nm}, 14 \mathrm{~nm}$, and 0.001 degrees in the $x, y$ and $\theta$ axes, respectively. These accuracies are measured using a double planes mirror SIOS SP 120 interferometer characterized by a high resolution of 0.1 nanometers. Otherwise, to reach these accuracies, the images are filtered using a $3 \times 3$ Median filter in order to reduce the acquisition noise of the CCD sensor. This filter is given by:

$$
M_{I}(p)=\operatorname{med}(\mathbf{I}(q) \mid q \subset \mathbf{W}(p))
$$

where $M_{I}$ is the image filtered such that for every pixel $p$, gray level $M_{I}(p)$ is the median of gray levels $\mathbf{I}(q)$ of $q$ pixels in the window $\mathbf{W}(p)$.

The acquisition noise (random image noise) can be shown

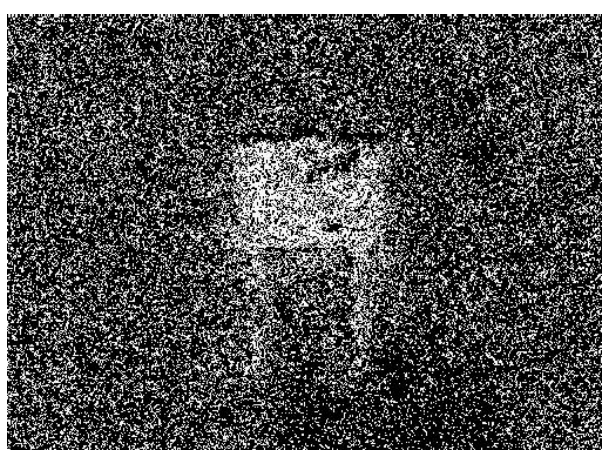

Fig. 4. Illustration of the acquisition noise of the CCD sensor used in the experimental validations.

in Figure 4. This noise image is reconstructed as follows:

$$
\begin{aligned}
& \mathbf{I}(x, y)-\mathbf{I}^{*}(x, y)=0, \text { the pixel is white, } \\
& \mathbf{I}(x, y)-\mathbf{I}^{*}(x, y) \neq 0, \text { the pixel is black, else }
\end{aligned}
$$

This means that the images $\mathbf{I}$ and $\mathbf{I}^{*}$ represent the same image at the initial position without any displacements, and in stable conditions of illuminations. In an ideal case, Figure 4 should be uniformly white. However, as it can be noticed it is not the case. This is a source of imprecision in the repositioning process and the Median filter is a solution to this issue.

\section{B. Validation with the addition of other Objects during the Process}

The proposed vision-based control law has been tested in order to measure its robustness in terms of accuracy and convergence in non-perfect conditions. The first test consists in the addition of another micro-object during the visual servoing achievement (positioning process). These micro-objects are not present in the desired image but in all images acquired during the positioning task. Figure 5 shows a sequence of images representing this test. Figure 5(a) illustrates the desired image of a single silicon micro-object, and Figures 5(b) to 5(i) show the error $\mathbf{I}-\mathbf{I}^{*}$ between the current image and the desired image. It can be noticed that in the current image there is a presence of additional micro-objects not present in the desired image. Changing the external conditions during the process, it can be seen that the quality of the convergence and the accuracy remain good. Figure 6 shows the platform velocities during this experiment.

\section{Validation using a Mask as a Desired Image}

The third experiment is based on the use of an image mask $\mathbf{I}_{m}$ as the desired image. Only the image of the object is considered and the background will not be considered in the desired image. This illustrates the fact that the object has not to be located at a specific position on the platform. The desired image is obtained as:

$$
\begin{aligned}
& \mathbf{I}^{*}{ }_{m}(x, y)=I^{*}(x, y), \text { if object }, \\
& \mathbf{I}^{*}{ }_{m}(x, y)=255, \text { else }
\end{aligned}
$$




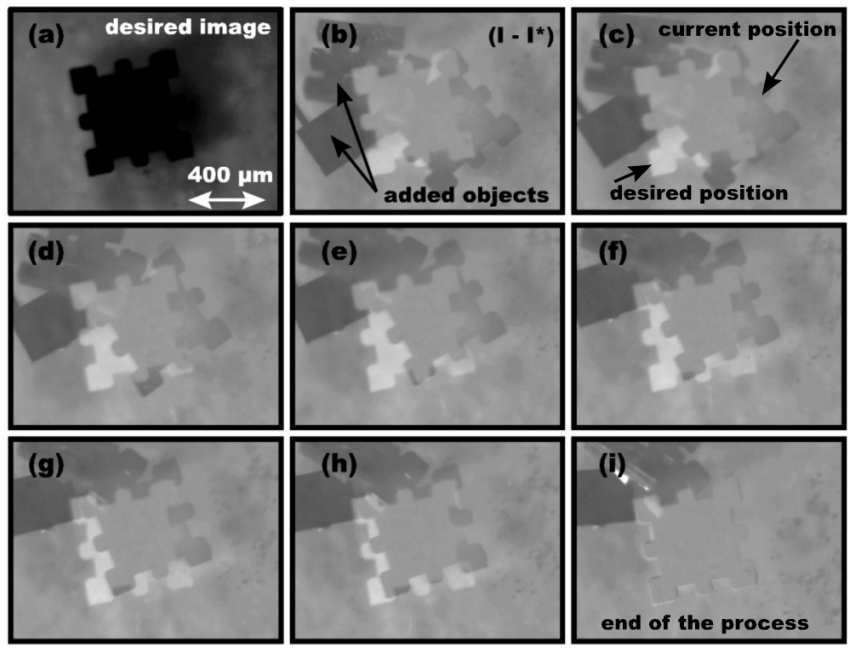

Fig. 5. (a) represents the initial position of the micro-object and (b) to (i) show the error $\left(\mathbf{I}-\mathbf{I}^{*}\right)$ between the current image $\mathbf{I}$ and the desired image $I^{*}$.

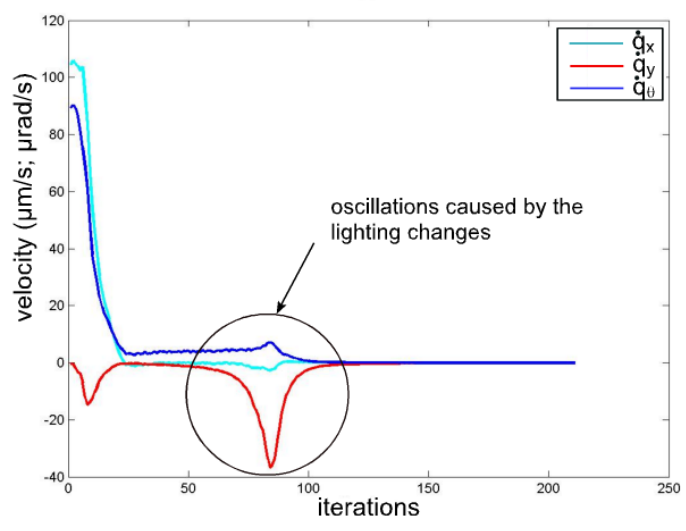

Fig. 6. Joint velocities components (in $\mu \mathrm{m} / \mathrm{s}$ and $\mu \mathrm{rad} / \mathrm{s}$ ) at each iteration of control scheme $\left(\dot{q}_{\mathbf{x}}, \dot{q}_{\mathbf{y}}\right.$ and $\left.\dot{\mathbf{q}}_{\theta}\right)$ for the experiment shown on Figure 5.

Figure 7 shows a sequence of images captured during the micropositioning process. Figure 7(a), represents the desired image which consists of the image of the micro-object inserted in a white image. Figures 7(b) to 7(g) represent the image error $\left(\mathbf{I}-\mathbf{I}^{*}{ }_{m}\right)$ during the visual servoing task.

Despite the presence of more than half of white pixels (virtual pixels) in the desired image $\mathbf{I}^{*}{ }_{m}$ which do not have corresponding pixels in the current image, the proposed control law remains robust and converges to zero as shown in Figure 8. By analyzing the behavior of the control law, we remark that there is a presence of small oscillations at the beginning because of the large number of pixels in the current image that have no correspondences in the desired image.

\section{Simultaneous Control of the Positioning Platform and the Optical Microscope Magnification}

The last experiment presented in this paper consists of the use of the proposed control law in multiple scale configuration. It means that the magnification (zoom) of the

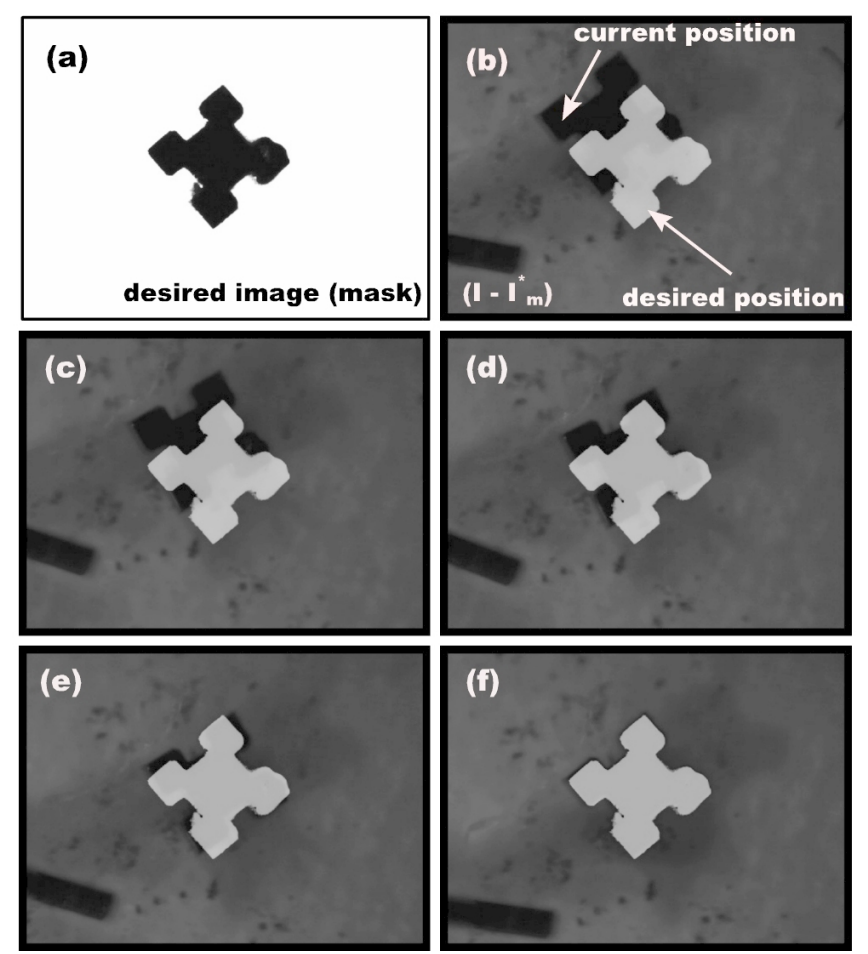

Fig. 7. Sequence image captured during the positioning process using a mask (see (a)) as a desired image.

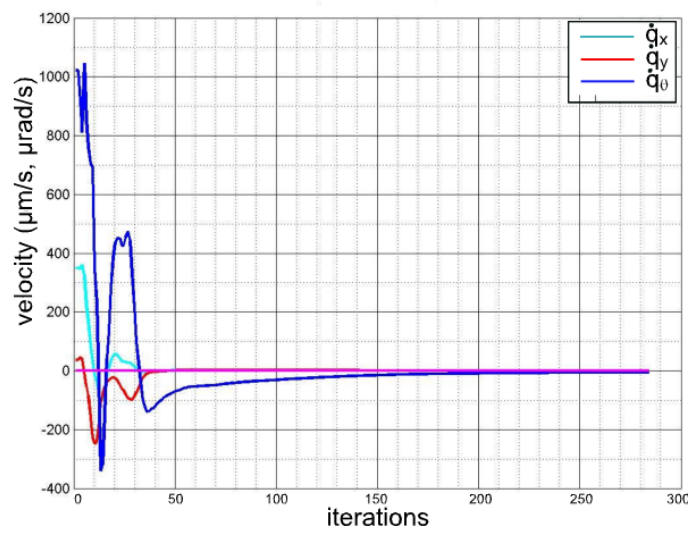

Fig. 8. Joint velocities components (in $\mu \mathrm{m} / \mathrm{s}$ and $\mu \mathrm{rad} / \mathrm{s}$ ) at each iteration of control scheme $\left(\dot{q}_{\mathbf{x}}, \dot{q}_{\mathbf{y}}\right.$ and $\left.\dot{q}_{\theta}\right)$ for experiment shown in Figure 7.
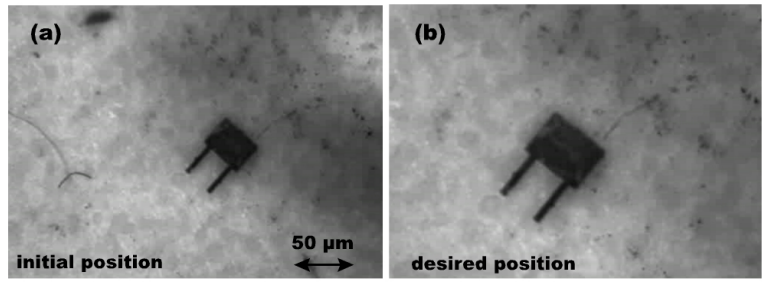

Fig. 9. (a) and (b) represent the initial position captured with a low magnification and the desired position acquired with a high magnification, respectively.

microscope is included in the control law. Thus, the idea is to use only a single view and then to resolve the problem of the limiting Field-of-View which characterizes the optical 
microscopes. To do this, the desired image is captured with a high magnification, i.e. $\times 9$ (low resolution/low Field-ofView) (Fig. 9(b)), and the visual servoing starts with a low magnification, i.e. $\times 2$ (high resolution/high Field-of-View) (see Fig. 9(a)). The control of the magnification consists of controlling the displacement of the optical microscope objective-lens along the optical axis. This latter is coaxial with the vertical axis $z$ which carried the rotation stage $\theta$ of the positioning platform. This allows to replace the expansive distributed imaging systems (i.e. low magnification camera for a global view of the scene and high magnification optical microscope for local view of the scene) which equipped most of the MEMS microassembly workcells.
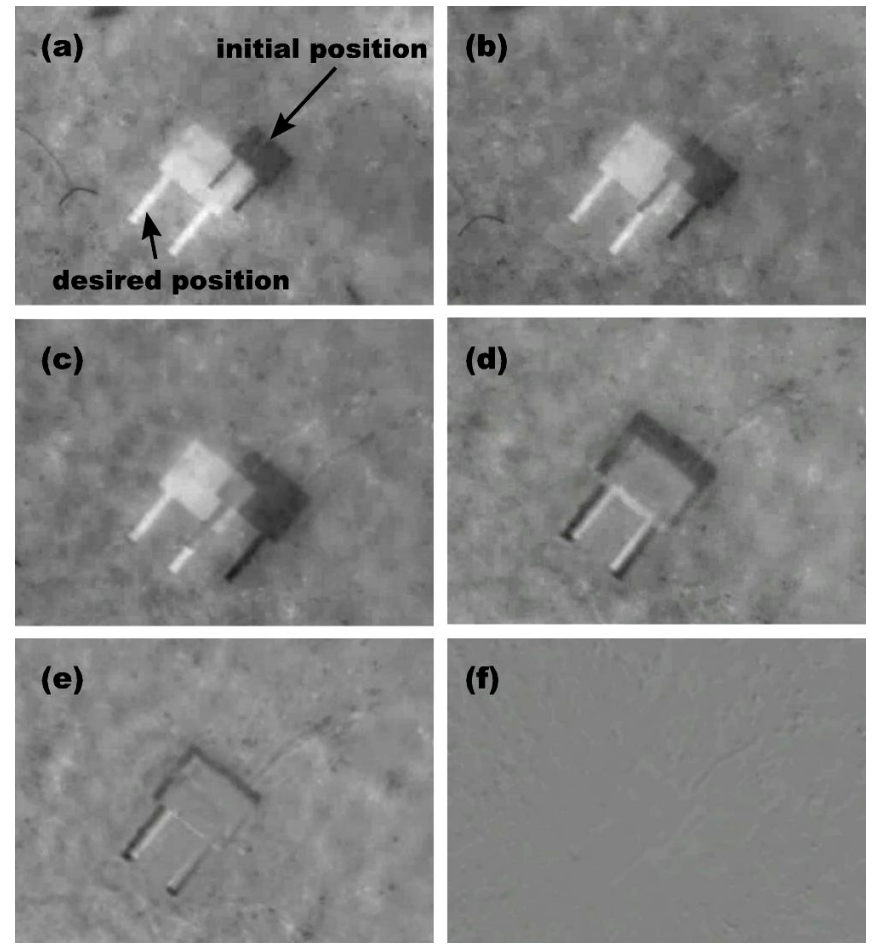

Fig. 10. Representation of the error $\left(\mathbf{I}-\mathbf{I}^{*}\right)$ during the positioning task and the magnification increasing in the same time.

Figure 10 illustrates the achievement of the multiple scale visual servoing. From Figure 10(a) to 10(d), it can be seen the error $\left(\mathbf{I}-\mathbf{I}^{*}\right)$ between the desired image and the current image as the evolution of the magnification function during the positioning task (see, also Fig. 11).

\section{CONCLUSION}

The problem of the automation of micropositioning tasks using a direct vision-based control approach was studied in this paper. Classical visual servoing schemes use geometrical visual features (points, lines, edges...) extracted and tracked in the images sequence. This kind of approaches require the use of image processing algorithms to extract these features from the images. It is very time consuming and complex to set up and often not robust to external conditions modification. Therefore, we have proposed a new control law based on the use of the pure image signal (pixels

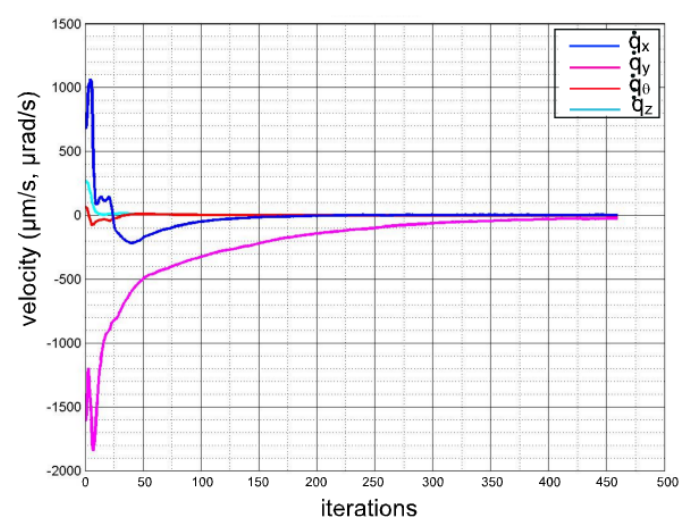

Fig. 11. Joint velocities components (in $\mu \mathrm{m} / \mathrm{s}$ and $\mu \mathrm{rad} / \mathrm{s}$ ) at each iteration of control scheme $\left(\dot{q}_{\mathbf{x}}, \mathbf{q}_{\mathbf{y}}, \dot{q}_{z}\right.$, and $\left.\dot{q}_{\theta}\right)$ for experiment shown on Figure 10 along with the motion of the camera zoom velocity.

intensity). This photometric approach has been tested on our microassembly workcell. From the different validation tests of the proposed control law, it appears that it is robust to global light variations, to important occlusions and to different perturbations. The proposed approach allows highly accurate positioning task accuracy (below 100 nanometers in translation and 0.001 degrees in rotation along the platform axis). This technique has also extended in order to include the control of the optical microscope magnification in visual servoing scheme. So, the first experimental results are promising in terms of accuracy and of the designed control law behavior. Future work will concern the use of these techniques to automate the complete MEMS assembly process which includes, positioning, orientation, picking, placing, and insertion of the different elements of the MEMS.

\section{REFERENCES}

[1] F. Chaumette and S. Hutchinson, Visual servo control, part 1: Basic approaches, IEEE Rob. and Aut. Mag. 13, (2006), no. 4, pp. 82-90.

[2] C. Collewet, E. Marchand, and F. Chaumette, Visual servoing set free from image processing, IEEE Int. Conf. on Rob. and Aut., (Pasadena, California), May 2008, pp. 81-86.

[3] A. Dame and E. Marchand, Entropy-based visual servoing, IEEE Int. Conf. on Rob. and Aut., (Kobe, Japan), 2009, pp. 707-713,

[4] B. Espiau, F. Chaumette, and P. Rives, A new approach to visual servoing in robotics, IEEE Trans. on Rob. and Aut., 8, (1992), no. 3, pp. 313-326.

[5] B.K.P. Horn and B.G. Schunck, Determining optical flow, Artificial Intelligence 17 (1981), no. 1-3, pp. 185-203.

[6] S. Hutchinson, G. Hager, and P. Corke, A tutorial on visual servo control, IEEE Trans. on Rob. and Aut., 12. 5, (1996), pp. 651-670.

[7] E. Marchand, Control camera and light source positions using image gradient information, IEEE Int. Conf. on Rob. and Aut., (Rome, Italy), 2007, pp. 417-422.

[8] M. Probst, R. Borer, and B. J. Nelson, A microassembly system for manufacturing hybrid mems, IFTOMM World Congress (Besançon, France), June 2007.

[9] S. Shet, V. R. Mehta, A. F. Fiory, M. P. Lepselter, and N. M. Ravindra, The magnetic field-assisted assembly of nanoscale semiconductor devices: A new technique, Jo. of Materials, 56(10), (2004), pp. 32-34.

[10] B. Tamadazte, E. Marchand, S. Dembélé, N. Le Fort-Piat. CAD model based tracking and 3D visual-based control for MEMS microassembly. The Int. J. of Rob. Research, IJRR, vol. 29, pp. 1416143, 2010.

[11] K. Yesin and B. Nelson, A CAD model based tracking system for visually guided microassembly, Robotica 23, (2005), pp. 409-418. 